\title{
Association of Bacteroides acidifaciens relative abundance with high-fibre diet- associated radiosensitisation
}

\author{
Chee Kin Then ${ }^{1+}$, Salome Paillas ${ }^{1 \dagger}$, Xuedan Wang ${ }^{2,3}$, Alix Hampson ${ }^{1}$ and Anne E. Kiltie ${ }^{1 *}$ (D)
}

\begin{abstract}
Background: Patients with pelvic malignancies often receive radiosensitising chemotherapy with radiotherapy to improve survival; however, this is at the expense of increased normal tissue toxicity, particularly in elderly patients. Here, we explore if an alternative, low-cost, and non-toxic approach can achieve radiosensitisation in mice transplanted with human bladder cancer cells. Other investigators have shown slower growth of transplanted tumours in mice fed high-fibre diets. We hypothesised that mice fed a high-fibre diet would have improved tumour control following ionising radiation (IR) and that this would be mediated through the gut microbiota.

Results: We investigated the effects of four different diets (low-fibre, soluble high-fibre, insoluble high-fibre, and mixed soluble/insoluble high-fibre diets) on tumour growth in immunodeficient mice implanted with human bladder cancer flank xenografts and treated with ionising radiation, simultaneously investigating the composition of their gut microbiomes by $16 \mathrm{~S}$ rRNA sequencing. A significantly higher relative abundance of Bacteroides acidifaciens was seen in the gut (faecal) microbiome of the soluble high-fibre group, and the soluble high-fibre diet resulted in delayed tumour growth after irradiation compared to the other groups. Within the soluble high-fibre group, responders to irradiation had significantly higher abundance of $B$. acidifaciens than non-responders. When all mice fed with different diets were pooled, an association was found between the survival time of mice and relative abundance of $B$. acidifaciens. The gut microbiome in responders was predicted to be enriched for carbohydrate metabolism pathways, and in vitro experiments on the transplanted human bladder cancer cell line suggested a role for microbial-generated short-chain fatty acids and/or other metabolites in the enhanced radiosensitivity of the tumour cells.
\end{abstract}

Conclusions: Soluble high-fibre diets sensitised tumour xenografts to irradiation, and this phenotype was associated with modification of the microbiome and positively correlated with B. acidifaciens abundance. Our findings might be exploitable for improving radiotherapy response in human patients.

Keywords: Gut microbiome, Dietary fibre, Inulin, Cellulose, Radiotherapy, Radiosensitisation, Pelvic tumour, B. Acidifaciens, Parabacteroides

\footnotetext{
*Correspondence: anne.kiltie@oncology.ox.ac.uk

${ }^{\dagger}$ Chee Kin Then and Salome Paillas contributed equally to this work. 'CRUK/MRC Oxford Institute for Radiation Oncology, Department of Oncology, University of Oxford, Old Road Campus Research Building, Off Roosevelt Drive, Oxford OX3 7DQ, UK

Full list of author information is available at the end of the article
}

(c) The Author(s). 2020, corrected publication 2021. Open Access This article is licensed under a Creative Commons Attribution 4.0 International License, which permits use, sharing, adaptation, distribution and reproduction in any medium or format, as long as you give appropriate credit to the original author(s) and the source, provide a link to the Creative Commons licence, and indicate if changes were made. The images or other third party material in this article are included in the article's Creative Commons licence, unless indicated otherwise in a credit line to the material. If material is not included in the article's Creative Commons licence and your intended use is not permitted by statutory regulation or exceeds the permitted use, you will need to obtain permission directly from the copyright holder. To view a copy of this licence, visit http://creativecommons.org/licenses/by/4.0/ The Creative Commons Public Domain Dedication waiver (http://creativecommons.org/publicdomain/zero/1.0/) applies to the data made available in this article, unless otherwise stated in a credit line to the data. 


\section{Background}

Patients with pelvic tumours, including bladder, cervix, and rectal cancers, who are receiving radiotherapy are often given additional radiosensitising chemotherapy to improve cure rates, at the expense of increased toxicity in local organs and tissues [1, 2]. With an ageing population, new approaches to radiosensitisation are urgently required. One such approach might be to modify the intake of dietary fibre by supplements before and during radiotherapy or current standard chemoradiation schedules, which would be a very cost-effective strategy, not expected to add to normal tissue toxicity [3, 4].

Wei et al. showed slower growth rates of subcutaneous lymphoma xenografts in mice fed a high-fibre diet (8\%) compared to mice on a low-fibre diet, with similar findings in both immune-deficient and immune-competent models [5]. This was associated with increased plasma and tumour butyrate levels, but the authors did not investigate the effects of the diet on the gut microbiome.

Dietary fibre manipulation can very rapidly alter the human gut microbiome, with changes in faecal short-chain fatty acid (SCFA) levels seen only 1 day after the diet reaches the distal gut [6]. Dietary fibre can also mediate systemic immune effects [7], as can the microbiota-derived SCFAs [8, 9]. Furthermore, dietary fibre structures align with phenotypes of specific microbes that differ in their metabolic pathways [10]. SCFAs are known to confer anticancer effects $[5,8,9]$. Other metabolites including small intermediate and end by-products of endogenous metabolic pathways, products of microbe-host co-metabolism, and exogenous signals arising from diet, drugs, and other environmental stimuli might also be important [11].

We hypothesised that systemic effects of altered metabolites secreted by gut bacteria on tumours, due to dietary fibre modification, could be exploited in conjunction with ionising radiation (IR) to achieve radiosensitisation.

The aims of this study were to examine the impact of the diet on the microbiome before and after irradiation and to correlate diet-induced microbiome changes with tumour growth and response to radiation treatment.

\section{Results}

The environmental microbiome had minimal impact on gut microbiome analysis

Female CD1 nude mice were injected subcutaneously with RT112 bladder carcinoma cells, and at the same time, they commenced a modified diet, namely one of the following: low dietary fibre $(0.2 \%$ cellulose $)$, low fibre with butyrate in drinking water, high soluble fibre $(10 \%$ inulin), high insoluble fibre ( $10 \%$ cellulose), and high mixed fibre (5\% cellulose, $5 \%$ inulin) (Fig. 1a and Additional file 1: Table S1). We quantified bacterial loads using PCR and gel electrophoresis, compared to known numbers of E. coli colony-forming units (CFUs). Our mouse samples contained more than $10^{4}$ bacterial CFUs which appeared to override contaminating species in the sample microbial communities (Fig. 1b). The PBS negative control was processed from the start of the DNA extraction identically to the luminal contents and tissue samples. The amount of nucleic acid detected in the PBS negative controls was extremely low, compared to that in the gut microbiota (Fig. 1c). Furthermore, the community microbiome in this negative control differed markedly from the gut microbiome of the mice (Fig. 1d). Therefore, the environmental microbiome had minimal impact on the analysis of the gut microbiomes of interest in this study.

The landscape, diversity, and enrichment of bacterial taxa in the gut microbiome samples collected when the tumours reached $50 \mathrm{~mm}^{3}$

In samples collected when the tumours reached $50 \mathrm{~mm}^{3}$ and $350 \mathrm{~mm}^{3}$, the faecal (hereinafter referred to as "gut microbiome") and caecal microbiomes were found to have similar bacterial components (Additional file 1: Figure S1). In mice culled when their tumours reached $50 \mathrm{~mm}^{3}$, butyrate levels in the faeces, measured by high-performance liquid chromatography (HPLC), were found to be in the millimolar range and were generally higher in the low fibre with butyrate and high soluble fibre groups ( $p=\mathrm{NS}$; Additional file 1: Figure S2A). The mean time for tumours to reach $50 \mathrm{~mm}^{3}$ was $12.8 \pm 1.4$ days (Additional file 1 : Figure S2B).

In faeces collected from culled mice when the tumours reached $50 \mathrm{~mm}^{3}$, abundance analysis revealed the five bacterial taxa with the highest abundance were Bacteroides acidifaciens, Parabacteroides, Akkermansia muciniphila, Lachnospiraceae, and S24-7 (Fig. 2a). In terms of alpha diversity, the soluble high-fibre group had a lower Shannon's index $(p<0.001)$ (Fig. 2b). This could be due to the higher abundance of $B$. acidifaciens, which lowered the diversity within groups. In terms of beta diversity, principal coordinate analysis showed a notable cluster effect among different groups, which indicates that samples within groups were more similar to each other than to those from the other groups (Fig. 2c). This suggested that the gut microbiome was indeed modified in this study, which might be a diet-effect or a cageeffect (see later). Regarding the abundance of specific taxa in different diet groups (Fig. 2d), the high soluble fibre diet significantly increased $B$. acidifaciens abundance $(p<0.001)$; the low-fibre diet increased Parabacteroides abundance $(p<0.001)$, the low-fibre diet with added butyrate increased Akkermansia muciniphila abundance $(p<0.001)$, and the high mixed fibre diet increased Lachnospiraceae abundance $(p=0.005)$. 


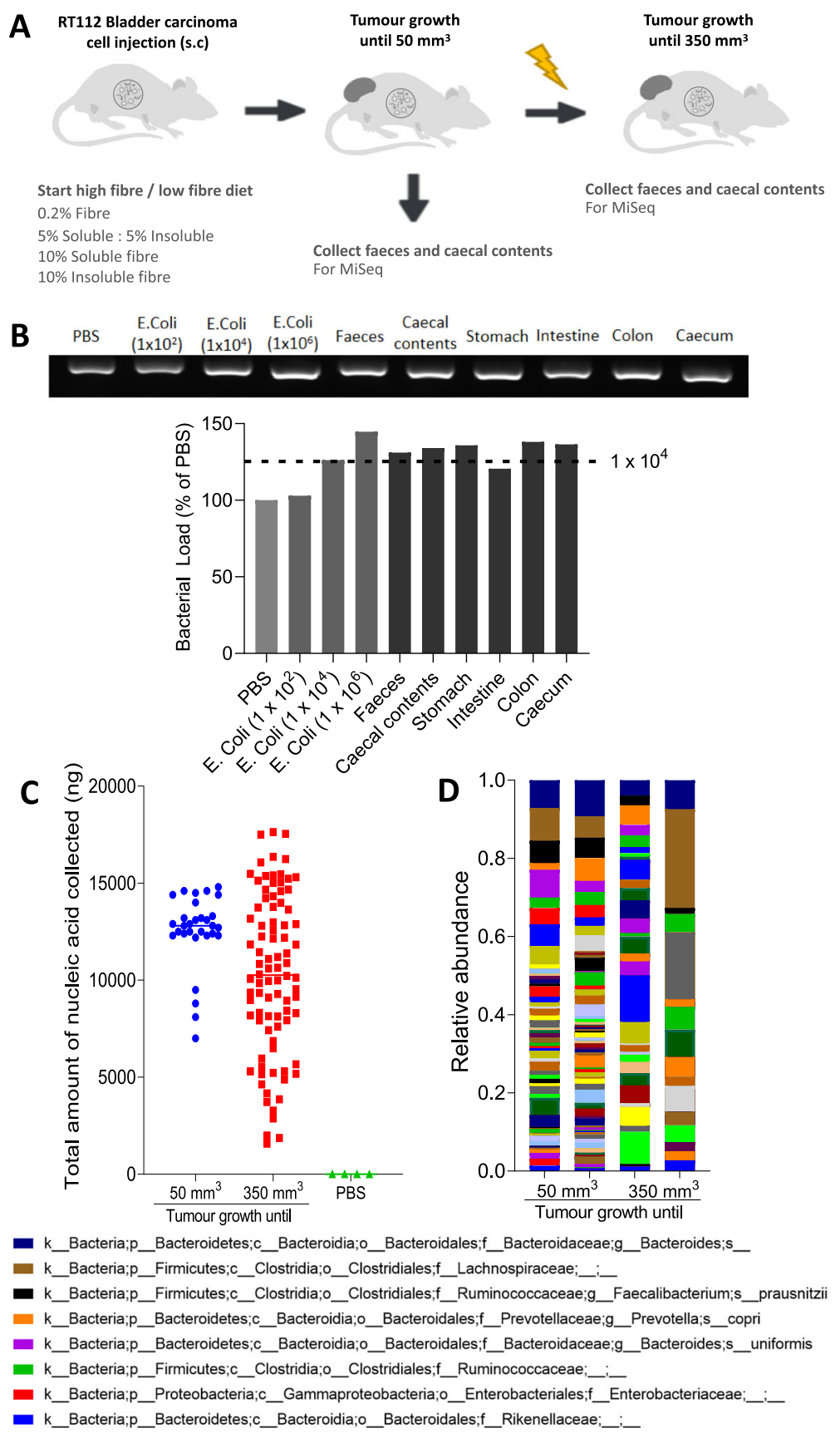

Fig. 1 The environmental microbiome had minimal impact on gut microbiome analysis. a Two microbiomes were analysed from the intestinal tract, namely faecal and caecal content samples collected when tumours reached $50 \mathrm{~mm}^{3}$ and $350 \mathrm{~mm}^{3}$, respectively. b Quantification of bacterial load from different tissue and luminal contents from mice, with E. coli $\left(1 \times 10^{2}, 1 \times 10^{4}, 1 \times 10^{6} \mathrm{CFUs}\right)$ as controls $(n=1$ mouse). c Comparison of the total amount of nucleic acid quantified by PicoGreen assay in all samples collected when the tumours reached $50 \mathrm{~mm}^{3}$ and $350 \mathrm{~mm}^{3}$. $\mathbf{d}$ Common bacterial taxa at the species level in 4 samples of PBS, as negative controls of DNA extraction by 165 rRNA sequencing

The landscape, diversity, and enrichment of bacterial taxa in the gut microbiome samples collected when the tumours reached $350 \mathrm{~mm}^{3}$

When the tumours reached $350 \mathrm{~mm}^{3}$, abundance analysis of the gut microbiome of both IR and non-IR cohorts revealed that the top 6 bacterial taxa with the highest abundance were S24-7, Akkermansia muciniphila, Bacteroides, Lachnospiraceae, Clostridiales, and B. acidifaciens (Fig. 3a). In terms of alpha diversity, the soluble HF group had a significantly lower Shannon's index $(p<0.001$ for the Kruskal-Wallis test) (Fig. 3b). In terms of beta diversity, principal coordinate analysis using the Bray-Curtis 


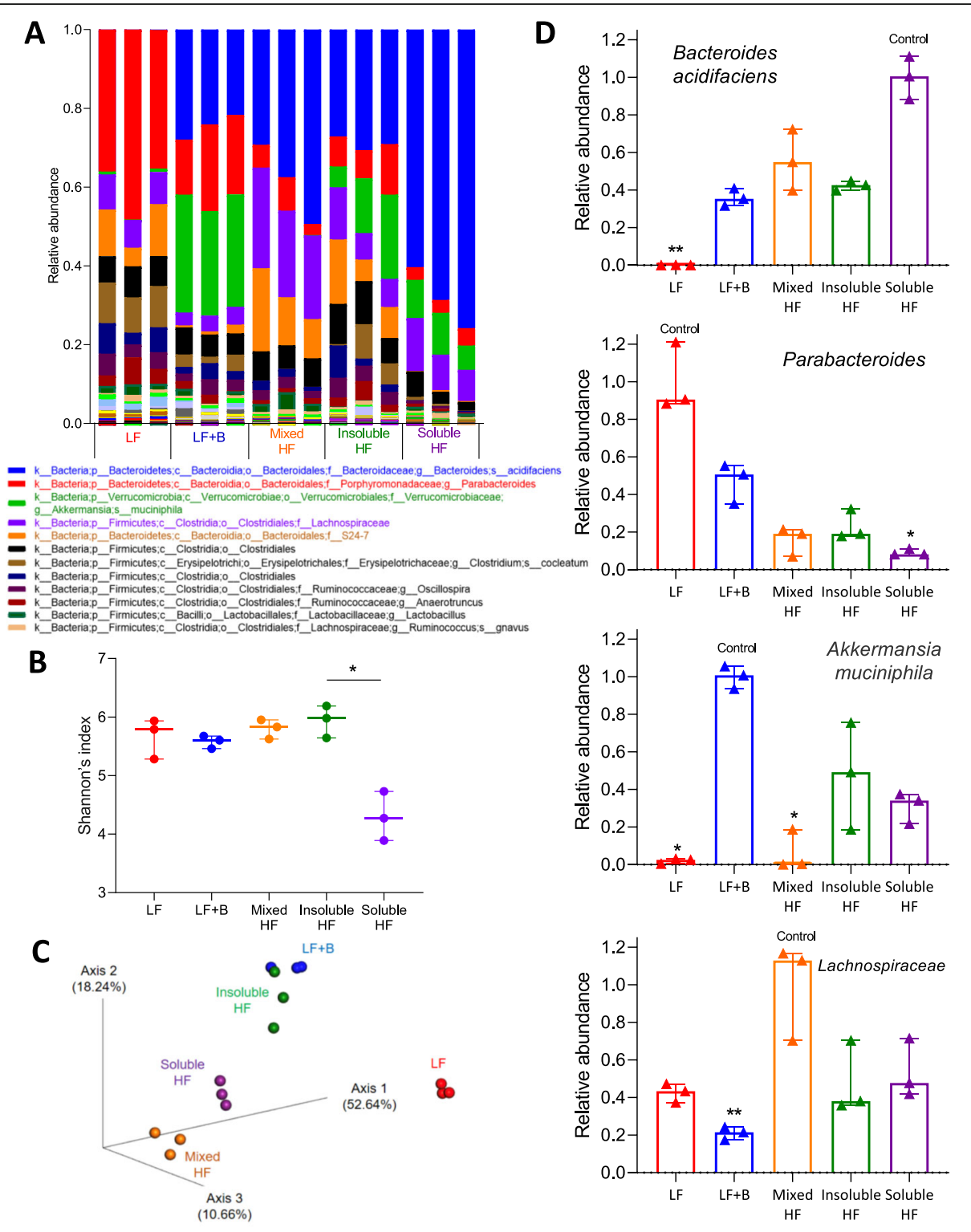

Fig. 2 Dietary fibre shapes the baseline gut microbiome when tumours reached $50 \mathrm{~mm}^{3}$. a Stacked bar plot of phylogenetic composition of common bacterial taxa at the species level when tumours reached $50 \mathrm{~mm}^{3}$. Faeces were collected from mice fed with low-fibre, low-fibre with butyrate, high mixed fibre, high insoluble fibre, and high soluble fibre diets ( $n=3$ for each group). $\mathbf{b}$ Shannon's index of gut microbiomes by the Kruskal-Wallis test. Error bars represent the interquartile range of diversity scores. c Principal coordinate analysis of gut microbiomes using the Bray-Curtis dissimilarity. A notable clustering effect by diet was seen in the gut microbiome. $\mathbf{d}$ Differentially abundant taxa when the tumours reached $50 \mathrm{~mm}^{3}$. All comparisons among different diet groups were performed by the Kruskal-Wallis test and Dunn's multiple comparison tests. All tests compared each median with the "control" denoted. The diet with the highest abundance of taxa was denoted as the control. ${ }^{*} p<0.05$; ${ }^{* *} p<0.01$; ${ }^{* *} p<0.001$

dissimilarity showed a notable clustering effect among different groups, which indicates that samples within groups were more similar to each other than to those from the other groups (Fig. 3c). The composition of the gut microbiome continued to evolve on the diets to the time the tumours reached $350 \mathrm{~mm}^{3}$, regardless of whether the mice were irradiated or not. The taxonomic cladogram of LEfSe (linear discriminant analysis effect size) of the gut microbiome showed that the high soluble fibre diet increased relative abundance of S24-7 (Additional file 1: Figure S3). B. acidifaciens, an acetate-producing bacterium [12, 13], was found to be of highest abundance in all but the LF diet group at $50 \mathrm{~mm}^{3}$, but by $350 \mathrm{~mm}^{3}$ was evenly distributed across the different diet groups, except the soluble HF group treated with radiation $(p=0.200$ for the Kruskal-Wallis test) (Fig. 3d).

Bacteroidales S24-7 was the highest abundance bacterial taxa in the second cohort. Its relative abundance was 


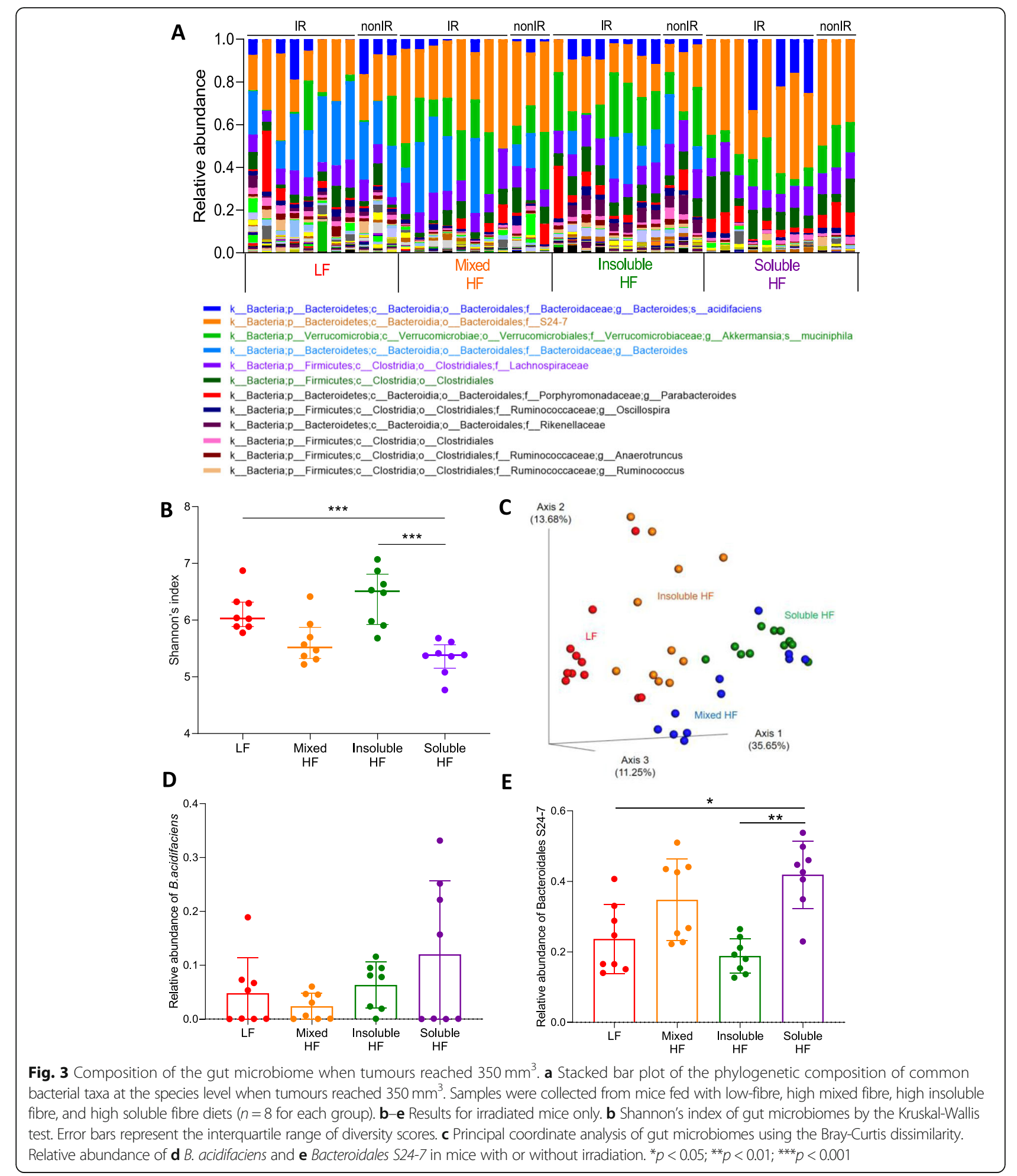

significantly higher in the mixed HF and soluble HF groups compared to the LF and insoluble HF groups $(p=0.001$ for the Kruskal-Wallis test) (Fig. 3e).
Soluble high fibre causes increased growth delay in irradiated bladder cancer cell xenografts

To investigate the effect of different diets on the tumour response in mice irradiated when the tumour had grown to $50 \mathrm{~mm}^{3}$, tumour growth was monitored to $350 \mathrm{~mm}^{3}$. 

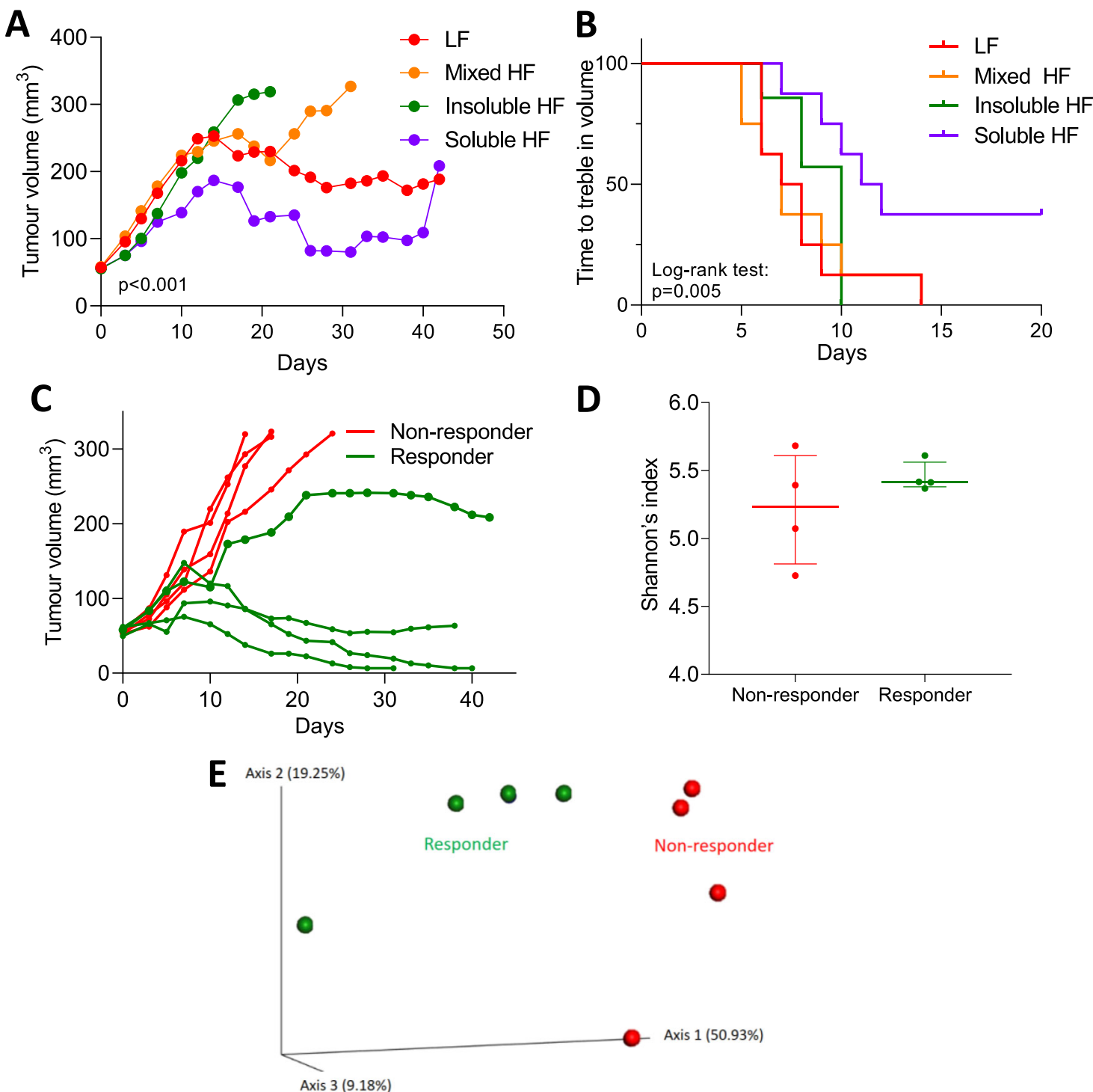

Fig. 4 Soluble high fibre causes increased growth delay in irradiated bladder cancer cell xenografts, and responses are influenced by gut microbiota composition. a Tumour growth in RT112 flank xenografts irradiated with 6 Gy IR, in mice fed low-fibre, high mixed fibre, high insoluble fibre, and high soluble fibre diets ( $n=8$ for each group). Tumour curve slopes were calculated by linear regression to represent tumour growth rates and compared by ANOVA. b The Kaplan-Meier survival curves for mice showing plots of time to treble tumour volume. c Mice in the soluble HF group were stratified into responders and non-responders based on tumour radiation response. $\mathbf{d}$ Shannon's index of gut microbiota in responders and non-responders by the Kruskal-Wallis test. Error bars represent the interquartile range of diversity scores. e Principal coordinate analysis of gut samples $(n=8)$ in the soluble HF group by response using the Bray-Curtis dissimilarity

Slopes of the tumour growth curves were obtained using linear regression to indicate the tumour progression rates (Fig. 4a). The high soluble fibre diet group had the slowest tumour growth rate. The slopes were $4.4 \pm 1.3$ for $\mathrm{LF}$, $16.1 \pm 1.7$ for mixed HF, $28.7 \pm 1.3$ for insoluble HF, and $0.4 \pm 1.5$ for soluble HF (Fig. $4 \mathrm{a}$ and Additional file 1: Figure S4 for individual irradiated mouse tumour growth curves). The Kaplan-Meier survival curves for time to treble tumour volume showed that the soluble HF group had the longest median survival time (7.5 days for LF, 7 days for mixed HF,
10 days for insoluble HF, 11.5 days for soluble HF; $p=$ 0.005, log-rank test) (Fig. 4b).

Among the eight mice fed the soluble high-fibre diet, four mice were classified as responders as, using linear regression, they had shallower slopes to the tumour growth curves, namely $7.3 \pm 1.2,-0.9 \pm 0.6,-4.8 \pm 0.5$, and $-5.2 \pm 0.9$. The other four mice were classified as non-responders with steeper slopes to the tumour growth curves, namely $34.6 \pm 3.0,31.1 \pm 2.6,23.3 \pm 1.0$, and $33.8 \pm 2.9$ (Fig. 4c). In terms of alpha diversity, there was no significant difference in Shannon's index between 
responders and non-responders (Fig. 4d). In terms of beta diversity, principal coordinate analysis of the BrayCurtis dissimilarity showed the gut microbiomes of responders and non-responders were more similar within groups than between groups (Fig. 4e).

\section{Differences in composition of the gut microbiome between responders and non-responders}

Linear discriminant analysis showed that mice responding to the soluble high-fibre diet with a slower tumour growth rate were enriched with Bacteroidaceae (f), Flavobacterium (g), Flavobacteriales (o), Lactococcus ( $g$ ), Streptococcus $(g)$, Streptococcaceae (f), Allobaculum ( $g$ ), and Erysipelotrichales $(o)$. The non-responding tumour-bearing mice were enriched with Bifidobacterium (g), Bidifobacteriaceae (f), Bifidobacteriales (o), Parabacteroides (g), Porphyromonadaceae (f), Lactobacillus (g), Lactobacillaceae (f), and Lactobacillales (Fig. 5a). In terms of effect size, B. acidifaciens $(s p)$ and Bacteroidaceae $(f)$ had the largest enrichment in responders, and Parabacteroides $(g)$ and Porphyromonadaceae ( $f$ ) had the largest enrichment in non-responders (Fig. 5b). To further explore these findings, the discrete false-discovery rates within all taxonomic levels were calculated (Fig. 5c). In responders, B. acidifaciens species and the Allobaculum genus and in non-responders Lactobacillus and Parabacteroides genera had $p$ values $<0.05$ The $B$. acidifaciens abundance was significantly higher in responders than that in non-responders ( $p=0.029$ (Fig. 5d), while the Bacteroidales S24-7 abundance was similar between responders and non-responders in the soluble $\mathrm{HF}$ group ( $p=0.200)$ (Fig. 5e).

\section{Metagenomics functional prediction of the gut microbiome by response in the soluble HF group and in vitro functional effects of short-chain fatty acids}

Functional prediction at KEGG (Kyoto Encyclopedia of Genes and Genomes) pathway level 2 revealed that the gut microbiome in responders was enriched for carbohydrate metabolism pathways and in non-responders for membrane transport pathways (Fig. 5f). For carbohydrate metabolism, the pathway with the highest level of enrichment, statistical analysis showed that responders had a significantly higher level than non-responders (Fig. 5g). Short-chain fatty acids (SCFAs), including acetate, propionate, and butyrate, are major products of fibre fermentation by the gut microbiota. We showed that all three SCFAs increased histone acetylation $(p=0.014$ for $10 \mathrm{mM}$ acetate, $p=0.004$ for $10 \mathrm{mM}$ propionate, $p<0.001$ for 10 $\mathrm{mM}$ butyrate; Fig. $5 \mathrm{~h}$ ) and tend to increase radiosensitivity ( $p=\mathrm{NS}$ for acetate, $p=\mathrm{NS}$ for propionate, $p=0.002$ for butyrate in 8 Gy; Fig. 5i) of RT112 bladder cancer cells. Single SCFAs reduced cell proliferation (Fig. 5j), while a physiological SCFA mixture conferred a stronger phenotype (Fig. 5j, purple bar) which was shown in a time-dependent pattern as well (Additional file 1: Figure S5A).

To validate the anti-tumoural effects of $B$. acidifaciens, we treated the bladder tumour cells with bacterial supernatants of $B$. acidifaciens and its cross-feeding with $L$. plantarum, and compared their effects with Bifidobacterium (acetateproducer) and L. plantarum (lactate-producer). Bacterial supernatants of $B$. acidifaciens and its cross-feeding with $L$. plantarum significantly increased cytotoxicity of bladder tumour cells compared to the other supernatants in day 2 (Fig. 5k) and in day 3 (Additional file 1: Figure S5B).

\section{Correlation between the abundance of $B$. acidifaciens or Parabacteroides genus and mouse survival time in IR and non-IR cohorts}

As $B$. acidifaciens was the "top hit" for responders and the Parabacteroides genus was one of the top two "hits" for non-responders in the soluble HF group, we explored how specific bacterial taxa affected mouse survival time. The correlation between $B$. acidifaciens abundance and time to culling was investigated across the diet groups. Some mice in the non-IR cohort lived as long as those in the IR cohort, i.e. $>40$ days, which may be a reflection of $6 \mathrm{~Gy}$ being a relatively low dose of radiation. In the IR cohort, the time of culling positively correlated with $B$. acidifaciens abundance $\left(R^{2}=0.528, p<0.001\right)$. However, a similar correlation was not seen in the non-IR cohort $\left(R^{2}=0.085, p=0.357\right)$ (Additional file 1: Figure S6A). Using the time for tumours to treble in volume as the outcome measure, mice with high $B$. acidifaciens abundance had a significantly prolonged median survival time (log-rank test, $p<0.001$ ) (Fig. 6a). A similar finding was seen in the IR cohort $(p=0.003)$, but not in the non-IR cohort $(p=0.236)$. In the IR cohort, the time to culling negatively correlated with the abundance of Parabacteroides genus (Additional file 1: Figure S6B; $R^{2}=0.164$, $p=0.022)$. However, a similar correlation was not seen in the non-IR cohort $\left(R^{2}=0.084, p=0.360\right)$. Mice in the low Parabacteroides genus abundance group had no significant difference in median time to treble in volume compared to the high abundance group (log-rank test, $p=0.374)$ (Fig. 6b). B. acidifaciens $(p=0.200$ for the Kruskal-Wallis test) and Parabacteroides genus ( $p=$ 0.005 for the Kruskal-Wallis test) abundance was evenly distributed among all cages, which suggested that the existence of these taxa in the gut microbiome was not a cage-specific effect (Additional file 1: Figure S7 and Additional file 1: Table S2).

\section{Discussion}

To date, the literature studying the effects of dietary fibre intake or manipulation of the gut microbiota on tumour growth is limited. Wei et al. showed that dietary 
A
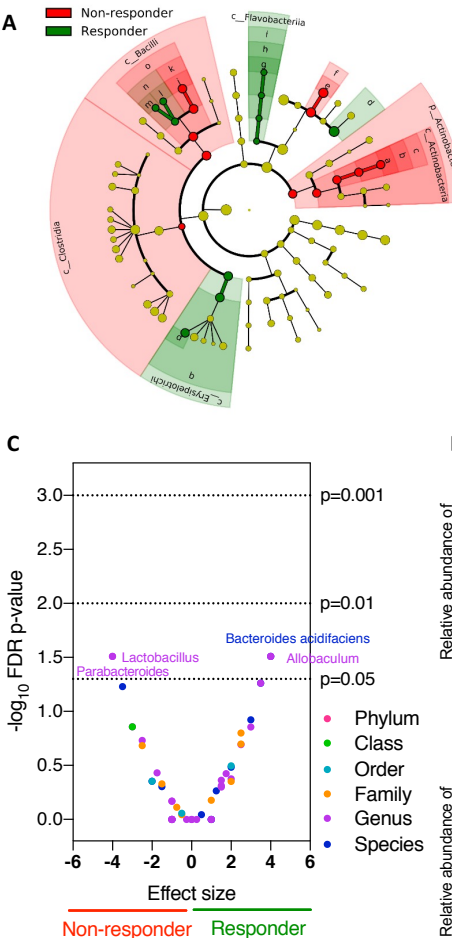

Non-responder Responder b: f_Bifidobacteriaceae c: o_Bifidobacteriales d: f_Bacteroidaceae e: g_Parabacteroides $f: f$ Porphyromonadacea g: 9_Flavobacterium i. Plavoacteriaces j: g_Lactobacillus b: f_Lactobacillacea I: g-Lactococcus m: g Streptococcus n: f - Streptococcace 0: o_Lactobacillales p: g_Allobaculum q: o_Erysipelotrichales a: g_Bifidobacterium

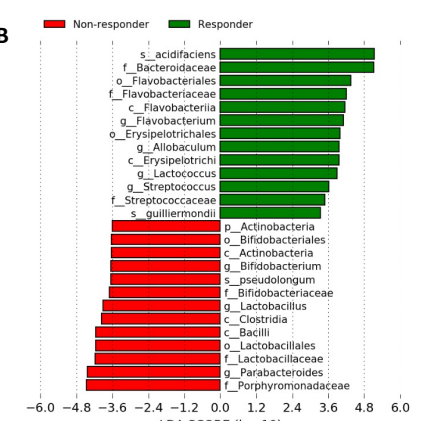

D

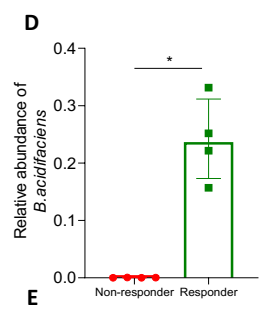

F
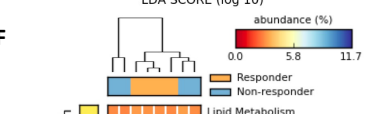
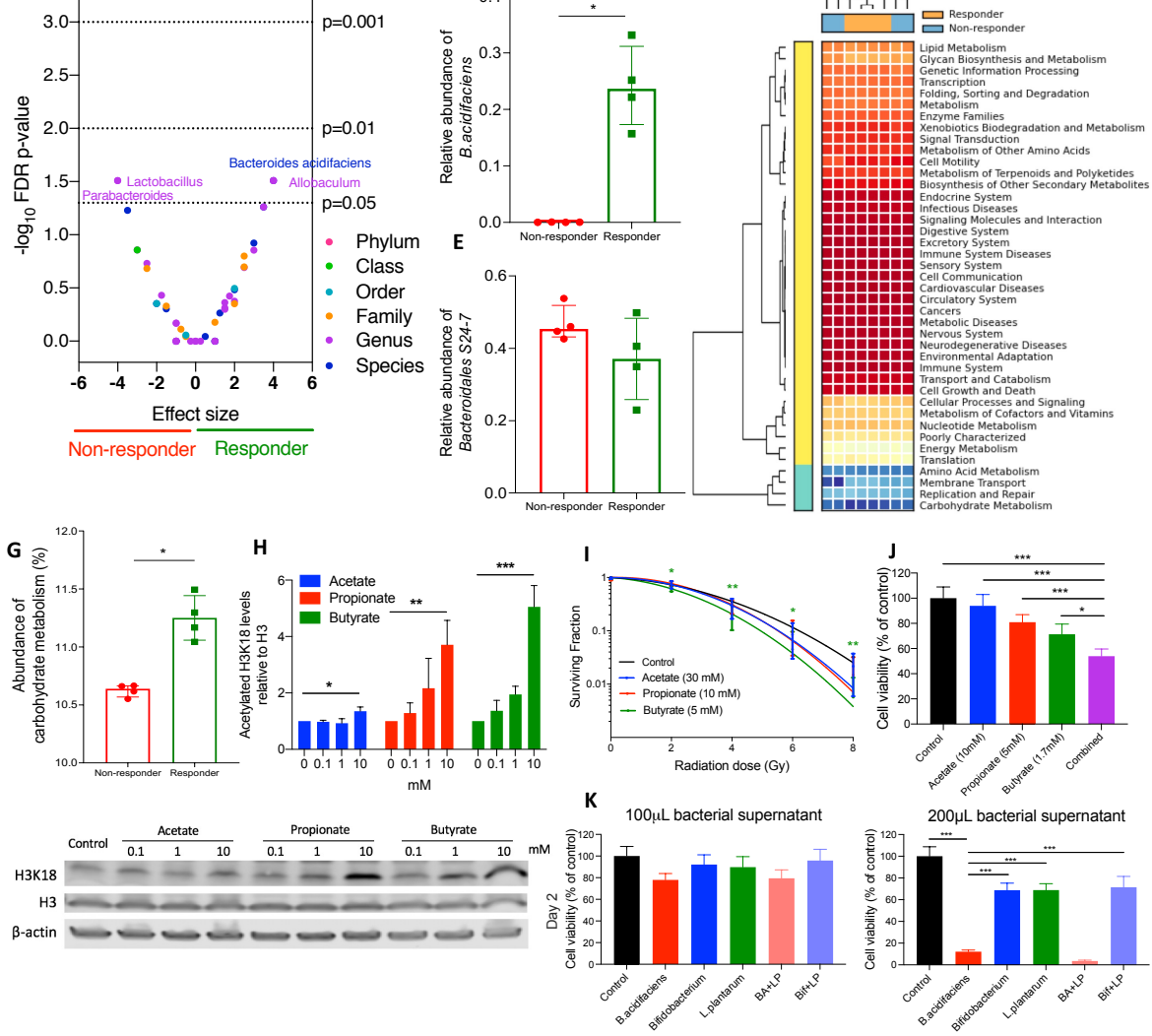

Fig. 5 Differences in composition of the gut microbiome between responders and non-responders. a Taxonomic cladogram from LEfSe showing differences among taxa between responders and non-responders in the soluble HF group. Dot size is proportional to the abundance of the taxon. b Linear discriminant analysis (LDA) scores computed for differentially abundant taxa in the microbiomes of responders (green) and nonresponders (red). Length indicates the effect size associated with a taxon, $p=0.05$ for the Kruskal-Wallis test. c Discrete false-discovery rate of different abundant taxa in responders and non-responders in the soluble HF group. Differential abundance within all taxonomic levels in responders versus non-responders by Mann-Whitney $U$ test. Dots are overlapping between Bacteroides acidifaciens and Allobaculum, and between Lactobacillus and Parabacteroides. Relative abundance of $\mathbf{d}$ B. acidifaciens and $\mathbf{e}$ Bacteroidales S24-7 and in responders and non-responders in the soluble HF group. $\mathbf{f}, \mathbf{g}$ Metagenomic functional prediction by PICRUSt of the gut microbiome in responders $(n=4)$ and non-responders $(n=4)$ in the soluble HF group with reference to the KEGG database level 2. Columns represent mice (responders, orange; non-responders, blue), and rows represent enrichment of predicted KEGG pathways (red, low enrichment; yellow, medium enrichment; blue, high enrichment). $\mathbf{h}$ Western blot analysis of histone acetylation levels of RT112 cells treated with SCFAs $(N=3)$. i Linear quadratic survival curves of IC10-treated RT112 cells with receiving irradiation of $0-8 \mathrm{~Gy}(\mathrm{~N}=3)$. $\mathbf{j}$ Cell survival analysis of RT112 cells treated with single SCFA and combined SCFAs mixture $(N=3)$. Combined (purple bar) denotes SCFA mixture of $10 \mathrm{mM}$ acetate, $5 \mathrm{mM}$ propionate, and $1.7 \mathrm{mM}$ butyrate. $\mathbf{k}$ Reduced cell survival of RT112 cells by bacterial supernatants at day $2(N=1)$. BA+FP denotes the cross-feeding of $B$. acidifaciens and L. plantarum, while Bif+FP denotes the cross-feeding of Bifidobacterium and L. plantarum. ${ }^{*} p<0.05 ;{ }^{* *} p<0.01 ;{ }^{* * *} p<0.001$ 


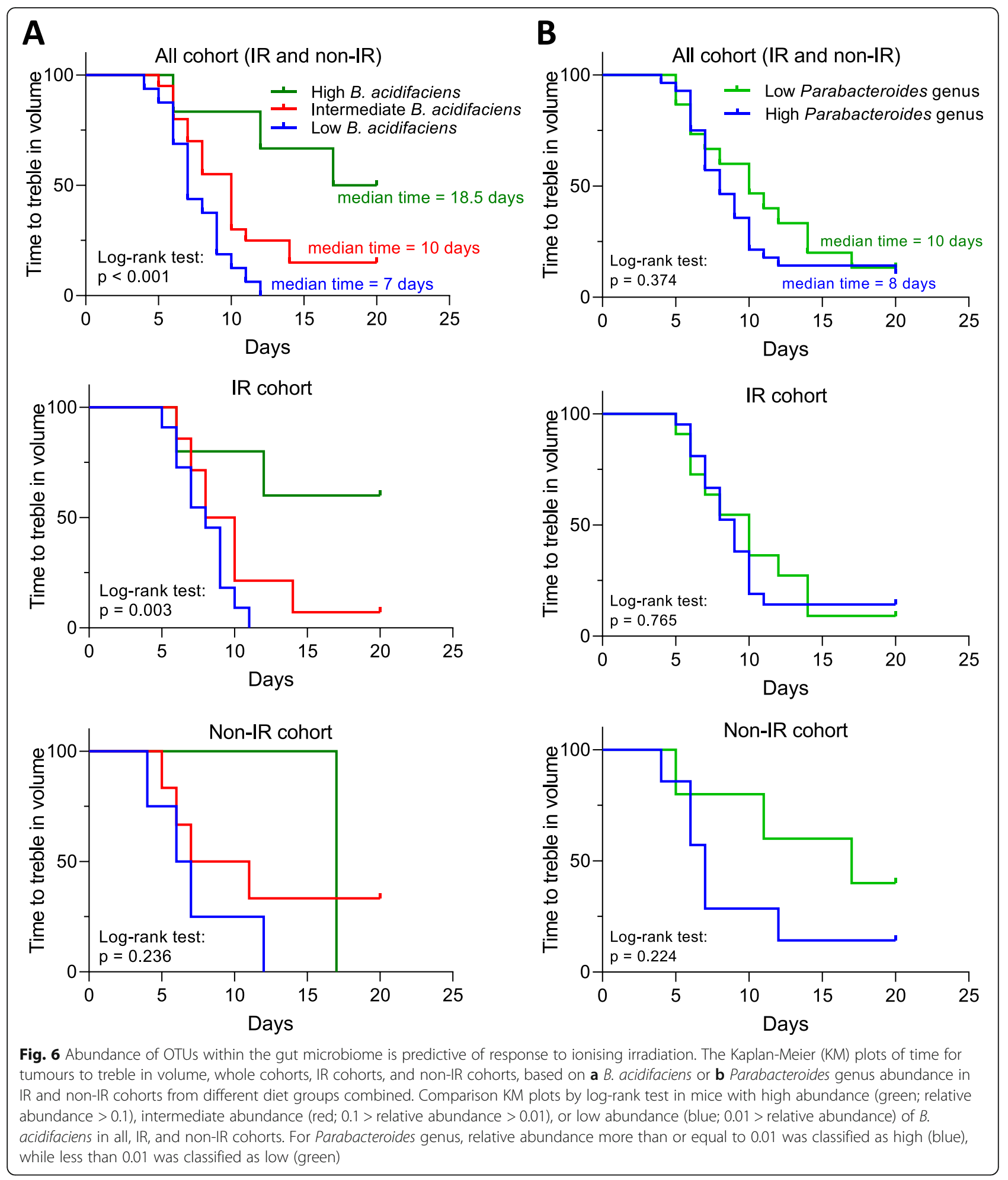

fibre and the associated butyrate production reduced subcutaneous lymphoma tumour growth with associated upregulation of histone 3 acetylation [5]. Hardman et al. found slower growth of breast cancer xenografts in mice fed with fish oil concentrate [14]. In contrast, Cougnoux et al. found that colibactin-producing E. coli enhanced tumour growth of colon cancer xenografts [15].

In this study, faecal and caecal microbiomes were investigated in mice fed the following: low-fibre diet, lowfibre diet with butyrate, high mixed fibre diet, high 
insoluble fibre (cellulose) diet, and high soluble fibre (inulin) diet, and profiles of both microbiomes were correlated. The gut microbiomes were shaped by the different modified diets within 2 weeks, and homogeneous gut microbiomes were seen in samples within groups. A distinct bacterial taxon was seen in each group: enrichment of $B$. acidifaciens in soluble HF, Parabacteroides in LF, Akkermansia muciniphila in LF with butyrate, and Lachnospiraceae in mixed HF.

The tumours were irradiated when they reached a volume of $50 \mathrm{~mm}^{3}$ (with no effect on diet on the time to reach this point) and monitored until they reached 350 $\mathrm{mm}^{3}$. During the course of the study, all mice developed an increased relative abundance of S24-7 family bacteria, thus indicating that their gut microbiomes had altered over the tumour growth period. Although the gut microbiomes became more heterogeneous, a notable cluster effect still existed in samples within groups. Mice responding to radiation in the soluble HF group were enriched with $B$. acidifaciens, and non-responding mice were enriched with the Parabacteroides genus.

A predictive metagenomics study of the gut microbiome in responders was enriched for carbohydrate metabolism pathways. This implies a higher level of fibre fermentation occurring in responders, reflecting the selection of bacteria more able to ferment carbohydrates. This could result in more SCFA production in the faeces. Both butyrate and propionate have been proposed to increase histone deacetylase inhibition [16], which is a known mechanism of cellular radiosensitisation $[17,18]$. In this study, we demonstrated that these phenotypes exist in bladder cancer cells (Fig. 5h, i). Furthermore, in vitro studies showed that SCFA reduced cell proliferation in liver cancer [19], induced apoptosis in lung cancer [20], and did both in breast cancer [21]. Vecchia et al. found that acetate and propionate potentiated the anti-cancer effects of butyrate in leukaemic, cervical adenocarcinoma, melanoma, and breast cancer cells [22]. We demonstrated similar findings in bladder cancer cells in this study (Fig. $5 \mathbf{j}$ and Additional file 1 : Figure S5A). Altogether, these data are supportive of carbohydrate metabolism or SCFA production being important in enhancing anti-cancer effects, including radiosensitisation.

When the mice from different diet groups were pooled, $B$. acidifaciens abundance was positively correlated with survival time, and mice with high $B$. acidifaciens had the longest median survival times using the Kaplan-Meier survival analysis. Bacteroidetes, including $B$. acidifaciens, have been proposed to produce the metabolic end products acetate, succinate, and possibly propionate, but not butyrate $[15,16]$. High acetate levels could act as a substrate for butyrate production, given that acetate is necessary for butyrate production, particularly in the butyryl-CoA:acetate CoA-transferase pathway [23, 24]. We speculate that the faecal butyrate levels could have been enhanced due to cross-feeding of butyrate-producing bacteria by $B$. acidifaciens. Proof-ofconcept was previously demonstrated for this by crossfeeding butyrate-producing bacteria (Faecalibacterium prausnitzii) and acetate-producing bacteria (Bifidobacterium adolescentis) [25]. Of note, Ramirez-Farias et al. showed that inulin increased both Faecalibacterium prausnitzii and Bifidobacterium adolescentis in a human volunteer study [26].

A modified gut microbiota can augment the efficacy of anti-tumoural treatment. However, most studies to date are limited to chemotherapy and immunotherapy, reviewed in [27, 28]. Recently, Herranz et al. found that depletion of gram-positive bacteria in the gut by vancomycin enhanced radiotherapy-induced anti-tumour immune response and further delayed tumour growth. However, the authors gave very large 21-Gy single fraction irradiation doses. Furthermore, reductions in abundance/absence of gram-negative bacteria (including Bacteroides and S24-7) were also seen, with increased Parabacteroides, which could be of significance. Here, we only gave a 6-Gy single fraction of IR which is more clinically relevant, and our findings were not immunologically mediated via $\mathrm{T}$ cells, as $\mathrm{CD} 1$ nude mice lack $\mathrm{T}$ cells.

To our knowledge, ours is the first study to provide evidence that a high soluble fibre diet, and its subsequent modification of the gut microbiome, can act to radiosensitise tumours.

Furthermore, B. acidifaciens was identified as a potential radiosensitiser because its abundance was enhanced by a high soluble fibre diet and positively correlated with tumour response to radiation and survival time in the IR cohort. This bacterium was first isolated in 2000 and was so named because it reduces the $\mathrm{pH}$ level of peptone-yeast broth with Fildes' digest [13]. Consistent with our findings, Marques et al. demonstrated that a high-fibre diet markedly increased the prevalence of $B$. acidifaciens [29]. Another study showed B. acidifaciens to be enriched in normal human subjects, compared to patients with inflammatory bowel disease [30], and $B$. acidifaciens increased insulin sensitivity and prevented further obesity [31]. However, the effect of these bacteria on tumour growth is still controversial. A study found increased $B$. acidifaciens abundance associated with hepatocellular carcinoma induced by a streptozotocin-high fat diet [32]. In contrast, $B$. acidifaciens reduces the isoflavone genisten, which is associated with increased risk of breast cancer [33], and B. acidifaciens was shown to contribute to the anti-tumour effect of medicinal Gynostemma saponins [34]. In this study, we revealed that bacterial supernatant from $B$. acidifaciens and its cross- 
feeding with $L$. plantarum caused significantly higher levels of cytotoxicity compared to the other supernatants (Fig. 5k and Additional file 1: Figure S5B). This result supports our finding that $B$. acidifaciens may drive the radiosensitising effect. Moreover, $B$. acidifaciens in vitro has a greater effect on cell kill than L. plantarum (lactate-producer; $p<0.001$ ), implying that metabolites other than lactate may be involved in its effect. Studies suggest that a broad range of gut microbiota-derived metabolites can enhance anti-tumoural effects or tumour response to anti-cancer treatments [35-37], so future work should include undertaking a global metabolomic analysis of B. acidifaciens-produced metabolites to identify radiosensitisers other than SCFAs with similar profiles.

We also found Bacteroidales S24-7 (or Candidatus Homeothermaceae [38] or Muribaculaceae [39]), an uncultured bacterium with limited characterisation, to be highly abundant in our study, especially in the mixed HF and soluble HF groups. The prevalence of this bacterium in humans is 20\% [38]. However, increased prevalence of Bacteroidales S24-7 of up to 70\% has been shown in the Hadza tribe of Tanzania who consume tubers containing large amounts of soluble fibre [38]. This indicates that high abundance of Bacteroidales S24-7 found in this study when the tumours reached $350 \mathrm{~mm}^{3}$ might be induced by soluble high fibre.

Environmental contamination is an inevitable issue in microbiome studies [40]. To minimise the influence of contamination in this study, bacterial loads of samples were quantified and appropriate negative controls were included. Bacterial loads from luminal contents and tissue samples contained more than $10^{4}$ CFUs which overrode the environmental bacteria communities. Furthermore, the bacterial compositions of the PBS negative controls were very different from those of the study groups, so the environmental microbiome was considered not to be a major source of bias in this study.

Although a strong correlation between $B$. acidifaciens abundance and tumour response to irradiation was seen, a limitation of this study is that only one cohort of mice was studied. Further studies are needed to determine the causal relationship between $B$. acidifaciens and radiosensitisation, and its underlying aetiology. This could be achieved by oral gavage of $B$. acidifaciens with or without other bacteria in gnotobiotic mice and in vitro culture studies of B. acidifaciens. As both gut bacteria [41] and irradiation [42] directly interact with the immune system, further in vivo studies need to be conducted in an immune-competent model to reveal how immunomodulation might contribute to the radiosensitisation.

\section{Conclusion}

A high soluble fibre diet increased responses of RT112 subcutaneous xenografts in CD1 nude mice to ionising radiation, and this phenotype was associated with higher relative abundance of $B$. acidifaciens. Possible mechanisms mediating this effect, which require further investigation, include the following: (1) increased concentrations of metabolites, including butyrate or other short-chain fatty acids in tumours, acting via HDAC inhibition or via other pathways; (2) suppression of overgrowth of unfavourable bacteria, such as Parabacteroides genus; and/or (3) enhancement of anti-tumoural immunity. Our findings suggest that dietary fibre modification and the resultant modification of the gut microbiome might be exploited to improve tumour responses to radiotherapy in human patients.

\section{Methods}

Mice and mouse diets

All animal work was done in accordance with UK Home Office Guidelines, following the ARRIVE (Animal Research: Reporting of In Vivo Experiments) guidelines, and approved by the University of Oxford Animal Welfare and Ethical Review Body (AWERB), under University of Oxford project licences P4B738A3B and P8484EDAE. Group sizes were chosen to detect large effect sizes by using a G-Power analysis program. All mice were purchased from Charles Rivers UK Ltd.

CD1 nude female mice at 6-7 weeks old were housed in a temperature-controlled environment with a $12-\mathrm{h}$ reversed-phase light/dark cycle (lights on 21:00 h) and provided with food and water ad libitum. These mice are immunodeficient, lacking a thymus, and therefore unable to produce $\mathrm{T}$ cells. Mice were randomised in Excel using the RAND function into four groups. At 7 to 8 weeks of age, mice were injected subcutaneously with RT112 bladder cancer cells (DSMZ, Germany) and started receiving either a low-fibre diet $(2 \mathrm{~g}$ cellulose $/ 3850 \mathrm{kcal})$, a high insoluble fibre diet $(100 \mathrm{~g}$ cellulose $/ 3850 \mathrm{kcal})$, a high soluble fibre diet $(100 \mathrm{~g}$ inulin $/ 3850 \mathrm{kcal})$, or a high mixed fibre diet $(50 \mathrm{~g}$ cellulose $+50 \mathrm{~g}$ inulin $/ 3850 \mathrm{kcal})$ for a maximum time of 9 weeks or until they were culled when the tumours reached $350 \mathrm{~mm}^{3}$. Faeces, caecal contents, and proximal and distal colons from the first cohort were taken when the tumour reached $50 \mathrm{~mm}^{3}$ (each group $n=3$ ) without irradiation to investigate the microbiome at baseline. Faeces and caecal contents from the second cohort were taken when the tumour reached $350 \mathrm{~mm}^{3}$ after IR (each group $n=8$ ) or without IR (each group $n=3$ ) or at the end of study (9 weeks after xenograft) to study the association between the gut microbiome composition and tumour response. 


\section{Xenograft model and irradiation method}

Mice were injected subcutaneously under anaesthesia into the right flank with $5 \times 10^{6}$ human bladder cancer cells (RT112) in RPMI medium (Sigma-Aldrich) with phenol red-free Matrigel (BD Biosciences) at a total volume of $100 \mu \mathrm{L}$ (1:1 ratio cell suspension to Matrigel). Tumour growth was measured three times a week, and size determined by callipers using length $\times$ width $\times$ height $\times \Pi / 6$. To assess the effects of different dietary fibres on tumour growth after irradiation in vivo, mice received ionising radiation to the tumour $(6 \mathrm{~Gy}$, single fraction, $300 \mathrm{kV}$, using a Gulmay-320 cabinet irradiator, Xstrahl Inc., UK). A dose of 6 Gy was chosen due to the promising effect in our previous radiosensitisation experiments with a HDAC inhibitor (manuscript in preparation.)

\section{Microbiome sample collection and DNA extraction}

All samples were transported on ice and kept at $-20^{\circ} \mathrm{C}$ for less than $2 \mathrm{~h}$ before DNA extraction. Bacterial genomic DNA was extracted using a DNeasy PowerSoil DNA Isolation Kit (QIAGEN Ltd., Manchester, UK), as per the Human Microbiome Project [43]. Briefly, by adding sodium dodecyl sulphate (SDS), microbial cells were lysed by mechanical disruption with a ceramic bead set on $3000 \mathrm{rpm}$ for $10 \mathrm{~min}$, followed by binding of DNA tightly to a silica membrane in a Spin Filter device at high salt concentrations. Eventually, DNA was collected into sterile elution buffer and quantified using a NanoDrop spectrophotometer. All DNA samples were kept at $-80^{\circ} \mathrm{C}$. All samples were collected and handled in sterile containers and equipment to minimise contamination. Those sent for sequencing (Omega Bioservices, Georgia, USA) were dried in an Eppendorf concentrator 5301 (Eppendorf North America Inc., USA) at a rotational speed of $1400 \mathrm{rpm}$ and centrifugal force of $240 \times \mathrm{g}$ for $1 \mathrm{~h}$ at $30^{\circ} \mathrm{C}$.

\section{Faecal butyrate level quantification}

Faecal samples were first homogenised in ice cold Millipore Synergy purified water. Thereafter, $20 \mu \mathrm{L}$ of sample or standard was taken and $10 \mu \mathrm{L}$ of internal standard (valeric acid, Alfa Aesar, UK) added prior to the addition of $5 \mu \mathrm{L} 15 \%$ percholoric acid. Samples were mixed and centrifuged at $12,000 \mathrm{~g}$ for $15 \mathrm{~min}$ at $4{ }^{\circ} \mathrm{C}$ followed by direct injection $(10 \mu \mathrm{L})$ of the supernatant. Highperformance liquid chromatography (HPLC) separation was carried out using a Waters Acquity H-Class Quarternary Solvent Manager with mobile phases of $0.1 \%$ formic acid in water (A) and methanol (B) and a gradient of $35-75 \%$ B on a Waters Acquity CSH C18, $1.7 \mu \mathrm{m}$, $100 \times 2.1$ column. Butyrate and internal standard (IS) were detected by mass spectrometry with a Waters Acquity TQ detector in positive electrospray ionisation mode. Butyrate was detected with a cone voltage of $20 \mathrm{~V}$ at selected ion recording (SIR) of $m / z 88.41(\mathrm{M}+\mathrm{H})$ and IS with a cone voltage of $15 \mathrm{~V}$ and SIR of $\mathrm{m} / z 103.2(\mathrm{M}+\mathrm{H})$.

\section{Cell line, drugs, and irradiator}

The RT112 bladder carcinoma cell line was obtained from the American Type Culture Collection. This cell line was cultured in RPMI-1640 medium (Sigma), supplemented with $10 \%$ foetal bovine serum (Invitrogen). Mycoplasma testing was negative. Sodium acetate, sodium propionate, and sodium butyrate were purchased from Sigma-Aldrich (Gillingham, UK) and used in $\mathrm{dH}_{2} \mathrm{O}$. Cells were irradiated in complete medium at a dose rate of $1.5 \mathrm{~Gy} / \mathrm{min}$ using a Gamma-Service Medical GmbH GSR D1 irradiator.

\section{Bacterial strain and its supernatant}

All bacterial strains were obtained from DSMZ-German collection of microorganisms. Three strains of bacteria, namely $B$. acidifaciens (BA; DSM 15896), Bifidobacterium animalis (Bif; DSM10140), and L. plantarum (LP), and two cross-feeding combinations $(B A+L P$ and $B i f+L P)$ were cultured in Gifu Anaerobic Broth, Modified (GAM; Nissui Pharmaceutical, Japan). Medium broth was prepared anaerobically in a Coy anaerobic chamber (Coylabs, US, N2 95\%, H2 5\%) and sealed into 10 -mL glass tubes before being autoclaved at $121^{\circ} \mathrm{C}$ for $15 \mathrm{~min}$. Bacteria $\left(10^{6} \mathrm{CFU} / \mathrm{mL}\right.$ starting population for each strain) were inoculated into these sealed tubes ascetically by injecting through a needle. They were then placed in a $37^{\circ} \mathrm{C}$ incubator for $24 \mathrm{~h}$ with constant shaking. Samples were centrifuged at $4000 \times g$ for $10 \mathrm{~min}$ to remove all particulate matter. Supernatants were then filtered through a $0.22-\mu \mathrm{m}$ polyethersulfone syringe filter (Millipore).

\section{Colony formation assay}

Cells were seeded in 6-well plates at appropriate densities in triplicate; treated with short-chain fatty acids, namely acetate, propionate, and butyrate, at appropriate concentrations for $24 \mathrm{~h}$; and irradiated with $0,2,4,6$, and $8 \mathrm{~Gy}$. After culturing for 10 days, colonies were fixed and stained with $0.5 \%$ crystal violet in $\mathrm{dH}_{2} \mathrm{O}$ and $20 \%$ methanol for $5 \mathrm{~min}$. Finally, they were quantified using a GelCount colony counter (Oxford Optronix). The surviving fraction was calculated by normalising the number of colonies for each condition to the unirradiated control.

\section{Cell survival analysis}

MTT 3-(4,5-dimethylthiazol-2-yl)-2,5-diphenyltetrazolium bromide assay was applied to assess cell viability by adding $0.25 \mathrm{mg} / \mathrm{mL}$ MTT (Life Technologies) to cells at $37^{\circ} \mathrm{C}$ for $1 \mathrm{~h}$ in the end of experiment. The absorbance 
at $595 \mathrm{~nm}$ of MTT-formazan was detected spectrophotometrically using POLARstar Omega Microplate Readers (BMG Labtech) after dissolution of the crystals in isopropanol. The percentage of cell viability was calculated by the formula: [Experimental group/Control group] $\times$ $100 \%$.

\section{Western blots}

Western blot samples were prepared as described in [44]. Protein was visualised using the following antibodies: H3K18Ac (Cell Signaling Technology, \#9675), H3 (Cell Signaling Technologies, \#4499S), and $\beta$-actin (Abcam, \#A1978), and an infrared LiCor Odyssey imaging system (LiCor Biosciences).

\section{Identification and quantification of bacterial DNA}

The microbiota of the contents of the intestinal tracts and the intestinal wall of the proximal and distal colon (tissue) was quantified by PCR of $16 \mathrm{~S}$ rRNA. This was performed on genomic DNA extracted as described above. The PCR was performed using primers-V3F (CCAGACTCCTACGGGAGGCAG) and V3R (CGTA TTACCGCGGCTGCTG) [45]. All primers were purchased from Sigma. For each sample, Phire Tissue Direct PCR Master Mix (Thermo Fisher Scientific) was used to amplify the $16 \mathrm{~S}$ rRNA gene hypervariable V3 region (product size $=200 \mathrm{bp}$ ). PCR amplifications were performed using the following conditions: $98^{\circ} \mathrm{C}$ for $5 \mathrm{~min}$ followed by 35 cycles at $98^{\circ} \mathrm{C}$ for $5 \mathrm{~s}$ each, $66.3^{\circ} \mathrm{C}$ for $5 \mathrm{~s}$, and $72{ }^{\circ} \mathrm{C}$ for $30 \mathrm{~s}$ and a final extension step at $72{ }^{\circ} \mathrm{C}$ for $1 \mathrm{~min}$. The amplification products were visualised on a $1 \%$ agarose gel after electrophoretic migration of $5 \mu \mathrm{L}$ of amplified material. A standard curve was created from serial dilutions of Escherichia coli from $1 \times 10^{2}, 1 \times 10^{4}$, and $1 \times 10^{6}$ colony-forming units (CFUs) which was quantified by CFU assay. All samples were run in duplicate. In CFU assay, $20 \mu \mathrm{L}$ of serial dilution of $E$. coli was incubated onto Luria-Bertani (LB) agar plates, and colonies were counted and bacterial concentrations of the original samples were estimated after $24 \mathrm{~h}$ incubation.

\section{Bacterial 16S rRNA gene sequencing}

16S rRNA gene sequencing methods were adapted from the methods developed for the NIH-Human Microbiome Project $[43,46]$. Raw $16 S$ rRNA reads and metadata have been made available in Figshare (https://figshare.com/ projects/The_gut_microbiota_may_drive_the_radiosensitising_effect_of_a_high_fibre_diet/68393) [47]. The amplification and sequencing of 16S rRNA gene V3V4 region were done commercially by Omega Bioservices (Georgia, USA) on a MiSeq platform (Illumina, Inc., San Diego, CA) using the $2 \times 300 \mathrm{bp}$ paired-end protocol, yielding paired-end reads with near-complete overlap. The primers containing adapters for Miseq sequencing were used for amplification and single-end barcodes, allowing pooling and direct sequencing of PCR products [48]. PBS negative controls were included to eliminate the confounding effects of environmental contamination.

All 16S rRNA gene-based metagenomic analysis was conducted using a QIIME2 platform [49]. Quality filtered sequences with $>97 \%$ identity were clustered into bins known as operational taxonomic units (OTUs), using open-reference OTU picking. The relative abundance of each OTU was obtained from all samples. In the taxonomic analysis, the microbiome at the phylum, class, order, family, genus, and species levels was classified with reference to the Greengenes database [50].

The analysis pipeline was as follows:

i. All sequences were trimmed to a length of 240, since the quality dropped above this length based on the sequence quality plots.

ii. De-noised sequencing errors by using the "Deblur" plugin in QIIME2 [51].

iii. Taxonomic assignment was performed with Greengenes [52] by the "feature-classifier" command.

iv. To visualise the differences in microbial composition between gut contents and tissue, a taxonomic profile was generated by conducting differential abundance analysis using balances in gneiss.

v. To identify the features characterising the differences between groups, the LEfSe method of analysis was performed to compare abundances of all bacterial clades [53]. By validation using the Kruskal-Wallis test at the $\alpha$ setting of 0.05 , effect size was obtained by LDA (linear discriminant analysis) based on the significantly different vectors resulting from the comparison of abundances between groups.

vi. To validate the significance of enrichment of bacterial taxa among different groups, discrete falsediscovery rates (DS-FDR) were calculated [54].

vii. A phylogenetic tree was generated by using the "phylogeny" plugin in QIIME2.

viii. To investigate the alpha and beta diversity, the diversity commands of "alpha-group-significance" and "beta-group-significance" were used to obtain Shannon's index and the Bray-Curtis dissimilarity. A principal coordinate ( $\mathrm{PCoA}$ ) plot was obtained by using the Emperor Tool based on the results of the Bray-Curtis dissimilarities.

ix. The OTU table was rarefied using the "alphararefraction" command in QIIME2. The alpha rarefraction plot showed the richness of the samples with increasing sequence count.

$x$. To predict the metagenome functional profiles, PICRUSt, a bioinformatics software package, was used to collapse predicted functions (KEGG 
Orthology; KO) based on 16S rRNA surveys into higher categories (KEGG pathway) after picking OTUs and normalisation [55].

\section{Statistics}

Power calculations for the number of mice per group were done using $G^{*}$ Power software version 3.1.9.4 [56]. Alpha diversity and relative abundance of specific bacterial taxa were compared using the Kruskal-Wallis test following by Dunn's multiple comparison test. All mice were classified into high, intermediate, or low diversity groups based on tertiles of distribution. Time to treble in volume was defined as the interval (in days) from the date of irradiation (growth to $50 \mathrm{~mm}^{3}$ ) to the date for the tumour to treble in volume. Tumour growth curves were analysed for each group, and their slopes were compared using one-way ANOVA. The LEfSe method of analysis was applied to determine the difference in bacterial taxa, using the Kruskal-Wallis test. Significantly different taxa presented from the previous comparison applying LEfSe method were used as input for LDA, which produced an LDA score. Volcano plots showed the significance of the taxa which are different among different groups, with $\log _{10}$ (FDR-adjusted $p$ values) on the $y$-axis and median-adjusted effect sizes on the $x$-axis. In addition, mice were also classified as having high, intermediate, and low abundance of $B$. acidifaciens or high and low abundance of Parabacteroides genus based on the relative abundance of these taxa in the gut microbiome sample. All analyses were conducted in QIIME2 and GraphPad Prism version 8.0 (La Jolla, CA).

All data in in vitro studies are representative of 3 independent biological replicates unless otherwise stated, with results shown as mean and standard deviations. One-way ANOVA with Dunnett's multiple comparison test was performed to analyse the data of Western blots and MTT assays. Two-way ANOVA with Dunnett's multiple comparison test was used to analyse the linear quadratic survival curves in colony formation assay.

\section{Supplementary information}

Supplementary information accompanies this paper at https://doi.org/10. 1186/s12915-020-00836-X.

Additional file 1: Figure S1. Similar bacterial components in the faecal and caecal microbiomes. Figure S2. Faecal butyrate levels and time taken for tumours to reach $50 \mathrm{~mm}^{\mathbf{3}}$. Figure $\mathbf{S 3}$. Differences in composition of the gut microbiome when tumours reached $350 \mathrm{~mm}^{\mathbf{3}}$ Figure S4. Individual mouse tumour growth curves. Figure S5. Cell survival analysis of RT112 bladder tumour cells treated with SCFAs and bacterial supernatants. Figure S6. Correlation of time to culling with $B$. acidifaciens or Parabacteroides genus abundance different groups. Figure S7. Effect of cage location of mice on relative abundance of $B$. acidifaciens and Parabacteroides genus. Table S1. Rodent diets used in the study with varying levels of cellulose or inulin per $4000 \mathrm{kcal}$. Table S2. Details mouse diets, cages, B. acidifaciens relative abundance and time of culling.

\section{Abbreviations}

ABC: ATP-binding cassette; B. acidifaciens: Bacteroides acidifaciens; BBN: NButyl-N-(4-hydroxybutyl)-nitrosamine; CFU: Colony formation unit; $E$. coli: Escherichia coli; FDA: False-discovery rate; Gy: Gray; HAT: Histone acetyltransferase; HDAC: Histone deacetylase; HF: High fibre; Insoluble HF: Insoluble high fibre; IR: Irradiation; KEGG: Kyoto Encyclopedia of Genes and Genomes; LC-MS: Liquid chromatography-mass spectrometry; LDA: Linear discriminant analysis; LEfSe: Linear discriminant analysis effect size; LF: Low fibre; LF+B: Low fibre plus butyrate; MIBC: Muscle-invasive bladder cancer; Mixed HF: Mixed high fibre; MTT: 3-(4,5-Dimethylthiazol-2-yl)2,5-diphenyltetrazolium bromide; OTU: Operational taxonomic unit;

PCoA: Principal coordinate analysis; PICRUSt: Phylogenetic Investigation of Communities by Reconstruction of Unobserved States; SCFA: Short-chain fatty acid; SDS: Sodium dodecyl sulphate; Soluble HF: Soluble high fibre

\section{Acknowledgements}

We thank Professor Simon Kroll and Dr. Anderson Ryan for their very helpful comments. We thank Dr. Jia-Yu Ke at Research Diets, Inc. for formulation of the mouse diets, Dr. Lisa Folkes for assistance with the faecal butyrate quantification, and Omega Bioservices (Georgia, USA) for the 16S rRNA gene sequencing on a MiSeq platform.

\section{Authors' contributions}

CKT extracted the DNA from mouse samples, performed the analysis and interpretation of the data, and drafted the manuscript. SP performed the animal experiments and collected the faeces, caecal contents, intestinal tissue, and blood. XW produced the bacterial supernatants. AH measured the faecal butyrate levels. AEK conceived the study, supervised the work, and revised the manuscript. All authors read and approved the final manuscript.

\section{Funding}

This work was funded by Cancer Research UK Programme grant C5255/ A23755 and Wellcome Trust Investigator Award 209397/Z/17/Z. The funding body had no role in the design of the study; in the collection, analysis, and interpretation of data; or in the writing of the manuscript.

\section{Availability of data and materials}

The datasets generated and/or analysed during the current study are available in the Figshare repository, https://figshare.com/projects/The_gut_ microbiota_may_drive_the_radiosensitising_effect_of_a_high_fibre_diet/683 93 [47].

Ethics approval and consent to participate

All animal protocols were approved by the University of Oxford Clinical Medicine Animal Welfare Ethics Review Board and conducted under animal project licences (PPL) P4B738A3B and P8484EDAE.

\section{Competing interests}

The authors declare that they have no competing interests.

\section{Author details}

${ }^{1}$ CRUK/MRC Oxford Institute for Radiation Oncology, Department of Oncology, University of Oxford, Old Road Campus Research Building, Off Roosevelt Drive, Oxford OX3 7DQ, UK. ${ }^{2}$ Department of Zoology, University of Oxford, Oxford, UK. ${ }^{3}$ Department of Biochemistry, University of Oxford, Oxford, UK.

Received: 21 February 2020 Accepted: 5 June 2020

Published online: 19 August 2020

References

1. Viswanathan AN, Lee LJ, Eswara JR, Horowitz NS, Konstantinopoulos PA, Mirabeau-Beale $\mathrm{KL}$, et al. Complications of pelvic radiation in patients treated for gynecologic malignancies. Cancer. 2014;120(24):3870-83.

2. Nishimura Y. Rationale for chemoradiotherapy. Int J Clin Oncol. 2004;9(6): 414-20.

3. Wedlake L, Shaw C, McNair H, Lalji A, Mohammed K, Klopper T, et al. Randomized controlled trial of dietary fiber for the prevention of radiationinduced gastrointestinal toxicity during pelvic radiotherapy. Am J Clin Nutr. 2017;106(3):849-57. 
4. Hedelin M, Skokic V, Wilderang U, Ahlin R, Bull C, Sjoberg F, et al. Intake of citrus fruits and vegetables and the intensity of defecation urgency syndrome among gynecological cancer survivors. PLoS One. 2019;14(1): e0208115.

5. Wei W, Sun W, Yu S, Yang Y, Ai L. Butyrate production from high-fiber diet protects against lymphoma tumor. Leuk Lymphoma. 2016;57(10):2401-8.

6. David LA, Maurice CF, Carmody RN, Gootenberg DB, Button JE, Wolfe BE, et al. Diet rapidly and reproducibly alters the human gut microbiome. Nature. 2014;505(7484):559-63.

7. Trompette A, Gollwitzer ES, Pattaroni C, Lopez-Mejia IC, Riva E, Pernot J, et al. Dietary fiber confers protection against flu by shaping Ly6c(-) patrolling monocyte hematopoiesis and CD8(+) T cell metabolism. Immunity. 2018;48(5):992

8. Bachem A, Makhlouf C, Binger KJ, de Souza DP, Tull D, Hochheiser K, et al. Microbiota-derived short-chain fatty acids promote the memory potential of antigen-activated CD8(+) T cells. Immunity. 2019;51(2):285-97 e5.

9. Kim M, Friesen L, Park J, Kim HM, Kim CH. Microbial metabolites, short-chain fatty acids, restrain tissue bacterial load, chronic inflammation, and associated cancer in the colon of mice. Eur J Immunol. 2018;48(7):1235-47.

10. Deehan EC, Yang C, Perez-Munoz ME, Nguyen NK, Cheng CC, Triador L, et al. Precision microbiome modulation with discrete dietary fiber structures directs short-chain fatty acid production. Cell Host Microbe. 2020;27(3):389404 e386.

11. Basson AR, Wijeyesekera A. Metabolic phenotyping for understanding the gut microbiome and host metabolic interplay. Emerg Top Life Sc. 2017;1 (4):325-32.

12. Macy JM, Probst I. The biology of gastrointestinal bacteroides. Annu Rev Microbiol. 1979;33:561-94.

13. Miyamoto $Y$, Itoh K. Bacteroides acidifaciens sp. nov., isolated from the caecum of mice. Int J Syst Evol Microbiol. 2000;50(Pt 1):145-8.

14. Hardman WE, Munoz J Jr, Cameron IL. Role of lipid peroxidation and antioxidant enzymes in omega 3 fatty acids induced suppression of breast cancer xenograft growth in mice. Cancer Cell Int. 2002;2(1):10.

15. Cougnoux A, Dalmasso G, Martinez R, Buc E, Delmas J, Gibold L, et al. Bacterial genotoxin colibactin promotes colon tumour growth by inducing a senescence-associated secretory phenotype. Gut. 2014;63(12):1932-42.

16. Silva LG, Ferguson BS, Avila AS, Faciola AP. Sodium propionate and sodium butyrate effects on histone deacetylase (HDAC) activity, histone acetylation, and inflammatory gene expression in bovine mammary epithelial cells. J Anim Sci. 2018;96(12):5244-52.

17. Donohoe DR, Collins LB, Wali A, Bigler R, Sun W, Bultman SJ. The Warburg effect dictates the mechanism of butyrate-mediated histone acetylation and cell proliferation. Mol Cell. 2012;48(4):612-26.

18. Groselj B, Sharma NL, Hamdy FC, Kerr M, Kiltie AE. Histone deacetylase inhibitors as radiosensitisers: effects on DNA damage signalling and repair. Br J Cancer. 2013;108(4):748-54.

19. Bindels LB, Porporato P, Dewulf EM, Verrax J, Neyrinck AM, Martin JC, et al. Gut microbiota-derived propionate reduces cancer cell proliferation in the liver. Br J Cancer. 2012;107(8):1337-44.

20. Kim K, Kwon O, Ryu TY, Jung CR, Kim J, Min JK, et al. Propionate of a microbiota metabolite induces cell apoptosis and cell cycle arrest in lung cancer. Mol Med Rep. 2019:20(2):1569-74.

21. Semaan J, El-Hakim S, Ibrahim JN, Safi R, Elnar AA, El Boustany C. Comparative effect of sodium butyrate and sodium propionate on proliferation, cell cycle and apoptosis in human breast cancer cells MCF-7. Breast Cancer. 2020;27(4):696-705

22. Vecchia MG, Carnelos Filho M, Fellipe CR, Curi R, Newsholme EA. Acetate and propionate potentiate the antiproliferative effect of butyrate on RBL2H3 growth. Gen Pharmacol. 1997;29(5):725-8.

23. Vital M, Howe AC, Tiedje JM. Revealing the bacterial butyrate synthesis pathways by analyzing (meta) genomic data. MBio. 2014;5(2):e00889.

24. Vital M, Karch A, Pieper DH. Colonic butyrate-producing communities in humans: an overview using omics data. mSystems. 2017;2(6):e00130-17.

25. Rios-Covian D, Gueimonde M, Duncan SH, Flint HJ, de los Reyes-Gavilan CG. Enhanced butyrate formation by cross-feeding between Faecalibacterium prausnitzii and Bifidobacterium adolescentis. Fems Microbiol Lett. 2015; 362(21):fnv176

26. Ramirez-Farias C, Slezak K, Fuller Z, Duncan A, Holtrop G, Louis P. Effect of inulin on the human gut microbiota: stimulation of Bifidobacterium adolescentis and Faecalibacterium prausnitzii. Brit J Nutr. 2009;101(4):541-50.
27. Alexander JL, Wilson ID, Teare J, Marchesi JR, Nicholson JK, Kinross JM. Gut microbiota modulation of chemotherapy efficacy and toxicity. Nat Rev Gastroenterol Hepatol. 2017;14(6):356-65.

28. Gopalakrishnan V, Spencer CN, Nezi L, Reuben A, Andrews MC, Karpinets TV, et al. Gut microbiome modulates response to anti-PD-1 immunotherapy in melanoma patients. Science. 2018:359(6371):97-103.

29. Marques FZ, Nelson E, Chu PY, Horlock D, Fiedler A, Ziemann M, et al. Highfiber diet and acetate supplementation change the gut microbiota and prevent the development of hypertension and heart failure in hypertensive mice. Circulation. 2017;135(10):964-77.

30. Ott SJ, Musfeldt M, Wenderoth DF, Hampe J, Brant O, Folsch UR, et al. Reduction in diversity of the colonic mucosa associated bacterial microflora in patients with active inflammatory bowel disease. Gut. 2004;53(5):685-93.

31. Yang JY, Lee YS, Kim Y, Lee SH, Ryu S, Fukuda S, et al. Gut commensal Bacteroides acidifaciens prevents obesity and improves insulin sensitivity in mice. Mucosal Immunol. 2017;10(1):104-16.

32. Xie G, Wang X, Liu P, Wei R, Chen W, Rajani C, et al. Distinctly altered gut microbiota in the progression of liver disease. Oncotarget. 2016;7(15):19355-66

33. Renouf $\mathrm{M}$, Hendrich $\mathrm{S}$. Bacteroides uniformis is a putative bacterial species associated with the degradation of the isoflavone genistein in human feces. J Nutr. 2011:141(6):1120-6.

34. Chen L, Tai WC, Brar MS, Leung FC, Hsiao WL. Tumor grafting induces changes of gut microbiota in athymic nude mice in the presence and absence of medicinal Gynostemma saponins. PLoS One. 2015;10(5): e0126807.

35. Johnson CH, Spilker ME, Goetz L, Peterson SN, Siuzdak G. Metabolite and microbiome interplay in cancer immunotherapy. Cancer Res. 2016;76(21): 6146-52.

36. Chuah LO, Foo HL, Loh TC, Mohammed Alitheen NB, Yeap SK, Abdul Mutalib NE, et al. Postbiotic metabolites produced by Lactobacillus plantarum strains exert selective cytotoxicity effects on cancer cells. BMC Complement Altern Med. 2019;19(1):114.

37. Ma J, Sun L, Liu Y, Ren H, Shen Y, Bi F, et al. Alter between gut bacteria and blood metabolites and the anti-tumor effects of Faecalibacterium prausnitzil in breast cancer. BMC Microbiol. 2020;20(1):82

38. Ormerod KL, Wood DLA, Lachner N, Gellatly SL, Daly JN, Parsons JD, et al. Genomic characterization of the uncultured Bacteroidales family S24-7 inhabiting the guts of homeothermic animals. Microbiome. 2016;4(1):36.

39. Lagkouvardos I, Lesker TR, Hitch TCA, Galvez EJC, Smit N, Neuhaus K, et al. Sequence and cultivation study of Muribaculaceae reveals novel species, host preference, and functional potential of this yet undescribed family. Microbiome. 2019;7(1):28.

40. Eisenhofer R, Minich JJ, Marotz C, Cooper A, Knight R, Weyrich LS Contamination in low microbial biomass microbiome studies: issues and recommendations. Trends Microbiol. 2019:27(2):105-17.

41. Lazar V, Ditu LM, Pircalabioru GG, Gheorghe I, Curutiu C, Holban AM, et al. Aspects of gut microbiota and immune system interactions in infectious diseases, immunopathology, and cancer. Front Immunol. 2018;9:1830.

42. Carvalho HA, Villar RC. Radiotherapy and immune response: the systemic effects of a local treatment. Clinics (Sao Paulo). 2018;73(suppl 1):e557s.

43. Human Microbiome Project C. A framework for human microbiome research. Nature. 2012;486(7402):215-21.

44. Kerr M, Scott HE, Groselj B, Stratford MR, Karaszi K, Sharma NL, et al. Deoxycytidine kinase expression underpins response to gemcitabine in bladder cancer. Clin Cancer Res. 2014;20(21):5435-45.

45. Wang HY, Du PC, Li J, Zhang YY, Zhang W, Han N, et al. Comparative analysis of microbiome between accurately identified $16 \mathrm{~S}$ rDNA and quantified bacteria in simulated samples. J Med Microbiol. 2014;63:433-40

46. Human Microbiome Project C. Structure, function and diversity of the healthy human microbiome. Nature. 2012;486(7402):207-14.

47. Then CK, Paillas S, Kiltie AE. The gut microbiota may drive the radiosensitising effect of a high fibre diet. figshare. 2019. Available from: https://figshare.com/projects/The_gut_microbiota_may_drive_the_ radiosensitising_effect_of_a_high_fibre_diet/68393. [cited 21 May 2020].

48. Caporaso JG, Lauber CL, Walters WA, Berg-Lyons D, Huntley J, Fierer N, et al. Ultra-high-throughput microbial community analysis on the Illumina HiSeq and MiSeq platforms. ISME J. 2012;6(8):1621-4.

49. Hall M, Beiko RG. 16S rRNA gene analysis with QIIME2. Methods Mol Biol. 1849;2018:113-29.

50. Balvociute M, Huson DH. SILVA, RDP, Greengenes, NCBI and OTT - how do these taxonomies compare? BMC Genomics. 2017;18(Suppl 2):114. 
51. Nearing JT, Douglas GM, Comeau AM, Langille MGI. Denoising the Denoisers: an independent evaluation of microbiome sequence errorcorrection approaches. Peerj. 2018;6:e5364.

52. DeSantis TZ, Hugenholtz P, Larsen N, Rojas M, Brodie EL, Keller K, et al. Greengenes, a chimera-checked 165 rRNA gene database and workbench compatible with ARB. Appl Environ Microb. 2006;72(7):5069-72.

53. Segata N, Izard J, Waldron L, Gevers D, Miropolsky L, Garrett WS, et al. Metagenomic biomarker discovery and explanation. Genome Biology. 2011; 12(6):R60.

54. Jiang L, Amir A, Morton JT, Heller R, Arias-Castro E, Knight R. Discrete falsediscovery rate improves identification of differentially abundant microbes. mSystems. 2017;2(6):e00092-17.

55. Langille MG, Zaneveld J, Caporaso JG, McDonald D, Knights D, Reyes JA et al. Predictive functional profiling of microbial communities using $16 \mathrm{~S}$ rRNA marker gene sequences. Nat Biotechnol. 2013;31(9):814-21.

56. Faul F, Erdfelder E, Lang AG, Buchner A. G*Power 3: a flexible statistical power analysis program for the social, behavioral, and biomedical sciences. Behav Res Methods. 2007;39(2):175-91.

\section{Publisher's Note}

Springer Nature remains neutral with regard to jurisdictional claims in published maps and institutional affiliations.

Ready to submit your research? Choose BMC and benefit from:

- fast, convenient online submission

- thorough peer review by experienced researchers in your field

- rapid publication on acceptance

- support for research data, including large and complex data types

- gold Open Access which fosters wider collaboration and increased citations

- maximum visibility for your research: over $100 \mathrm{M}$ website views per year

At $\mathrm{BMC}$, research is always in progress.

Learn more biomedcentral.com/submissions 